\title{
Fabrication of an imaging diffraction grating for use in a MEMS-based optical microspectrograph
}

\author{
S Grabarnik, A Emadi, H Wu, G de Graaf, G Vdovin \\ and R F Wolffenbuttel \\ Faculty EEMCS, Department ME/EI, Delft University of Technology, Mekelweg 4, 2628 CD, Delft, \\ The Netherlands \\ E-mail: s.grabarnik@tudelft.nl
}

Received 12 December 2007, in final form 18 February 2008

Published 13 May 2008

Online at stacks.iop.org/JMM/18/064006

\begin{abstract}
The design, fabrication and performance of a highly miniaturized optical spectrometer are described. The volume of the opto-electronic system is only $50 \mathrm{~mm}^{3}$. The main components are a planar imaging diffraction grating and a commercially available CCD camera. System integration is based on MEMS technologies on a glass wafer. The imaging grating circumvents the need for collimating optics, while the planar grating design is essential for limiting fabrication complexity and enabling assembly of the optical and electronic parts. The system consists of two glass plates placed in parallel with well-defined spacing and comprises all spectrometer components including slit and diffraction grating. The glass plates were fabricated using a single glass wafer with standard lithography applied. The spectrometer demonstrated a $6 \mathrm{~nm}$ FWHM spectral resolution in an operating range from $600 \mathrm{~nm}$ to $700 \mathrm{~nm}$.
\end{abstract}

(Some figures in this article are in colour only in the electronic version)

\section{Introduction}

IC-compatible optical microspectrometers fabricated using microelectromechanical system (MEMS) technology in silicon have huge potential in applications where either extreme requirements on size or weight (such as space), or low costs per unit in high volume with a user-friendly interface (such as point-of-care medical diagnostics using a disposable chemical analysis microsystem) are decisive. The benefits of a miniaturized opto-electronic system include the integration of optics with microelectronic circuits, at the expense, however, of moderate optical specifications [1]. Microspectrometers based on optical resonance (mostly Fabry-Perot) and diffraction gratings have been demonstrated. Although resonator-based devices have found application in channel separation for optical communication, grating-based MEMS systems have proven more suitable for operation over a wide spectral range. To date, mainly lensless MEMS-based systems with a simple transmission grating that yield limited resolution have been realized. The technological constraints of IC- compatible processing limited the maximum spectral resolving power of those systems to $R_{\max }=\lambda / \Delta \lambda \sim 20[2,3]$.

The most effective approach to circumvent this limitation is to design a microspectrometer based on an a-spherical concave diffraction grating. However, such a device is difficult to combine with planar IC technology. A compromise lies in a flat imaging diffraction grating, which is presented in this paper. Due to the imaging properties of such a grating it is possible to combine collimating, dispersion and focusing in one single optical element, thus simplifying the spectrometer design considerably. Moreover, planar imaging gratings allow for the low cost per unit IC-compatible fabrication and thus are well suited for high-volume production.

Imaging gratings have already been implemented in waveguide-based systems and in compact classical types of spectrometers [4-8]. The reported devices have a volume typically in the $1 \mathrm{~cm}^{3}$ range with a $5 \mathrm{~nm}$ resolution over a $100 \mathrm{~nm}$ spectral range. The objective of this work is to design and fabricate a microspectrometer providing the best possible spectral resolution at demanding dimensional and technological constraints. The dimensions are to be reduced 
by an order of magnitude (to about $50 \mathrm{~mm}^{3}$ ) by applying MEMS technologies, while optical performance should not be sacrificed. Moreover, system integration should be used to avoid laborious alignment procedures.

Available analytical models of the diffraction gratingbased imaging systems (see for example [9]) have been modified to meet the constraints in the design of a microspectrometer. The obtained model takes into account such factors as optical aberrations and diffraction effects and allows the estimation of the best achievable spectral resolution of a spectrometer. It was used to find the optimal parameters of the design as a compromise between size and resolution, hence minimizing spectrometer dimensions at given specifications.

The performance of a spectrometer critically depends on the alignment of components in the optical system. As a solution to this problem, the planar spectrometer design was introduced, which is composed of two parallel glass plates and mounted directly on the surface of an imaging sensor. All spectrometer components, including input slit and diffraction grating, were fabricated on one plane wafer. Thus the slit and the grating are automatically aligned. The tolerances in the alignment of the two glass plates after dicing are not strict: $15 \mu \mathrm{m}$ for the spacing and $100 \mu \mathrm{m}$ lateral misalignment of the glass plates, which highly simplifies the assembly of the device.

The fabricated device was mounted directly on top of an image sensor, takes a volume of only $50 \mathrm{~mm}^{3}$ and features a $6 \mathrm{~nm}$ spectral resolution in a $100 \mathrm{~nm}$ operating range from $600 \mathrm{~nm}$ to $700 \mathrm{~nm}$. The planar concept presented in this paper opens a way for the design of a completely integrated silicon microspectrometer with an input slit, diffraction grating and an array of photodetectors integrated on the same chip.

\section{Planar spectrometer design}

The theory of an imaging grating is presented for example in [10] and [11]. Figure 1(a) illustrates the geometry of a diffraction grating which images point $A$ into point $B$. Plane $y z$ of the coordinate system $x y z$ lies in the grating plane and the origin of the coordinate system coincides with the grating central point $\mathrm{O}$. Consider point $\mathrm{O}$ and a point $P(y, z)$ somewhere in the grating plain. Then the optical path difference between light beams passing from $A$ to $B$ via point $P$ and via point $O$ is

$$
F(\lambda, y, z)=A P B-A O B+m \lambda N(y, z),
$$

where $N(y, z)$ is a function equal to the number of the grating grooves between the points $P(y, z)$ and $O, m$ is a diffraction order and $\lambda$ is a wavelength. Equation (1) can be decomposed into power series:

$$
\begin{aligned}
F(\lambda, y, z) & =\sum_{i=0}^{\infty} \sum_{j=0}^{\infty} F_{i j} y^{i} z^{j} \\
& =\sum_{i=o}^{\infty} \sum_{j=0}^{\infty}\left(M_{i j}+m \lambda N_{i j}\right) y^{i} z^{j}
\end{aligned}
$$

A path difference $F$ in equation (2) equal to zero corresponds to the aberration-free convergent wavefront. (a)
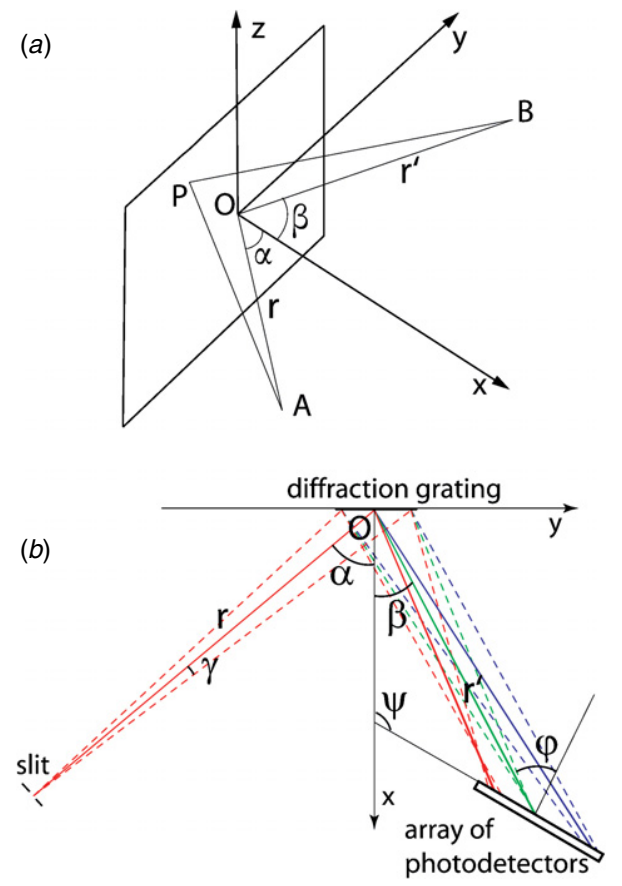

Figure 1. A grating imaging point A into point B (a) and general spectrometer layout $(b)$.

Thus, the coefficients $F_{i j}$ determining the deviation of the wavefront proportional to $y^{i} z^{j}$ can be considered as the aberration coefficients. The coefficients $M_{i j}$ depend on the system configuration and the coefficients $N_{i j}$ characterize the grating groove pattern [11]. After decomposing the function $N(y, z)$ into a series, one can obtain

$$
N(y, z)=\sum_{i=0}^{\infty} \sum_{j=0}^{\infty} N_{i j} y^{i} z^{j}
$$

Setting a function $N(y, z)$ equal to a number $N_{g}$, the shape of the corresponding groove can be found as a function of $y(z)$ by solving equation (3) relative to $y$. For the desired wavelength $\lambda_{0}$ the coefficients $N_{i j}$ can be selected in such a way that the aberration coefficients, $F_{i j}$, are zero, thus yielding an aberration-minimum image at a point A. Given the geometrical parameters of the system, the grating coefficients can be calculated analytically:

$N_{i j}=-\frac{M_{i j}}{m \lambda_{0}}=\frac{1}{i ! j ! m \lambda_{0}}\left[\frac{\partial^{i+j}(A P B-A O B)}{\partial y^{i} \partial z^{j}}\right]_{(0,0)}$

The exact formulas for $M_{i j}$ are listed for example in [11]. The local period of a grating along the $o y$-axis is $d=$ $(\partial N / \partial y)^{-1}$. The period $d$ at the central grating point $y=$ $z=0$ is referred to as the grating period $d_{0}$. The parameter $\lambda_{0}$ in equation (3) is the design wavelength, which is the central wavelength of the spectrometer operating bandwidth. It is clear from equation (2) that aberrations at the wavelength $\lambda$ become larger as the difference $\lambda-\lambda_{0}$ increases. The angular deviation of an image produced by a ray diffracted at the point $P(y, z)$ from the ideal image point $\mathrm{B}$ (figure $1(a)$ ) is given by the partial derivatives of the path difference function, $\frac{\partial F}{\partial y}$ and $\frac{\partial F}{\partial z}$. 


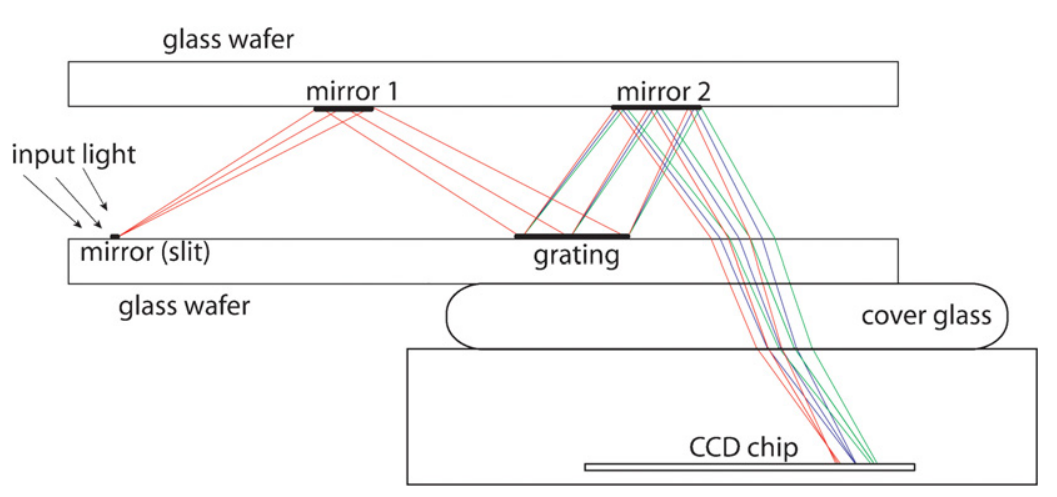

Figure 2. Planar spectrometer design.

Such a deviation defines the aberration-induced broadening of the image of the source point A. Since the minimal spectral range $\Delta \lambda$ resolved by a spectrometer is proportional to the width of the image of the entrance slit, the best resolution can be expected at wavelengths close to $\lambda_{0}$.

Equations (1)-(4) were used for the analytical calculation of the contribution of aberrations to the dependence of the spectrometer resolution on the design parameters. The general layout of an imaging grating-based spectrometer is presented in figure $1(b)$.

It is characterized by the distances $r$ and $r^{\prime}$ from the grating to the slit and the image plane, respectively, input aperture angle $\gamma$, the angle $\psi$ determining position of the image sensor, incident angle $\alpha$ and diffraction angle $\beta$ for the wavelength $\lambda_{0}$. The spectral resolution of a spectrometer presented in figure $1(b)$ depends on the width of the entrance slit, on the diffraction broadening of the slit image, which can be estimated as $r^{\prime} 2 \lambda / D$ with $D$ the width of the illuminated part of the grating and on the aberrations introduced by the grating. All those contributions determine the size of the entrance slit image and have been calculated to give the analytical dependence of the spectrometer resolution on the dimensions and configuration of the device, grating period and operating spectral bandwidth. In a planar design with two opposing members in parallel $\psi=\pi / 2$. The grating operates in the first diffraction order.

The grating period should be minimized to achieve best spectral resolution, but for a lithographically fabricated grating the grating period $d$ is limited by the lithography resolution and is two times larger than the minimum feature size. Taking into account the available lithography resolution the grating period in the model was set to $2 \mu \mathrm{m}$. As a compromise between moderate spectral resolution and reasonable throughput the input aperture angle was selected to be $0.05 \mathrm{rad}$. Simulations indicate that it is possible to achieve resolution of about 5-7 nm with the size of $r=r^{\prime}$ as small as $6-8 \mathrm{~mm}$, and increasing the dimensions of the device would not considerably improve the resolution. Thus, $6-8 \mathrm{~mm}$ is the optimal size of the microspectrometer and the starting point of the design. The operating range of the spectrometer was selected to be from $600 \mathrm{~nm}$ to $700 \mathrm{~nm}$ and the wavelength of $650 \mathrm{~nm}$ is the design wavelength (see equations (1)-(4)).

The schematic of spectrometer design is shown in figure 2. The spectrometer consists of two laterally aligned glass plates, which are parallel to each other. All spectrometer optical components, including the input slit and the diffraction grating, work in reflection. The light from a source is reflected by a metal strip, acting as a slit, and redirected toward the diffraction grating fabricated on the lower plate by mirror 1 in the upper glass plate. The diffracted light is reflected by mirror 2 and is finally projected on the imager through the lower glass plate.

The contour of mirror 1 defines the input aperture of the spectrometer, since the non-reflected light escapes the system through the glass plate. As a rule, the cross-section of the light beam propagating in an optical system is circular to avoid undesired diffraction effects. Thus the contours of the grating and mirrors were designed elliptical taking into account the light incident angle.

The distance from the grating to the photosensor (the distance $r^{\prime}$ in figure 1) was $6.4 \mathrm{~mm}$ and the distance between two parallel plates was $1.5 \mathrm{~mm}$, making the design very compact. The planar design with two opposing members in parallel implies $\psi=90^{\circ}$, which is not optimal. Better resolution can be achieved with higher values of $\psi$. However, increasing $\psi$ also increases the angle at which light falls on the photosensor (angle $\varphi$ in figure 1). This angle should not exceed some maximum value to avoid Fresnel reflection (from the surfaces of the cover glass and silicone chip) of a substantial part of radiation. Limiting the angle $\varphi$ by the device dimensions to $55^{\circ}$ causes the feasible resolution of the planar design (and $\psi=90^{\circ}$ ) to be reduced by $20 \%$ as compared to the case of rotated planar members. However the simplicity of this design greatly compensates for this inferior optical performance. Higher incident angles (angle $\alpha$ in figure 1) would yield a better spectral resolution, but there is a practical limit. The amount of light reflected from the glass surface increases with the angle of incidence due to Fresnel reflection. Such parasitic reflections contribute to the stray light as is discussed below. As a compromise the angle of incidence was set at $60^{\circ}$.

The model has been used to calculate a feasible spectral resolution of $14 \mathrm{~nm}$ over the entire band (from $600 \mathrm{~nm}$ to $700 \mathrm{~nm}$ ). The resolution in terms of the width of a monochromatic line at half maximum (FWHM) results as $7 \mathrm{~nm}$. In the central part of the operating bandwidth (in the range from $635 \mathrm{~nm}$ to $665 \mathrm{~nm}$ ), the resolution is about 
$3 \mathrm{~nm}$. The theoretical simulation and optimization of the design parameters do predict the feasibility of a miniature device with the volume more than an order of magnitude smaller than state-of-the-art reported in the literature [5-8]. Moreover, the reduced volume is not at the expense of inferior optical performance. Finally, the proposed planar design with integrated slit and diffraction grating on the same chip provides a key for a simple assembly process. Therefore, actual fabrication in a MEMS technology was pursued.

The parameters of the designed spectrometer were used to calculate the grating structure using equation (4) and to generate the pattern for the lithography mask. The grating structure is symmetrical with respect to the $y$-axis (figure 1) and consists of a number of curved grooves. The distance between adjacent grooves decreases with increasing $y$ so the grating pattern resembles the off-center part of a Fresnel lens. The local period of the grating varies from $1.5 \mu \mathrm{m}$ to $2.8 \mu \mathrm{m}$ along the grating surface, which was within the limits of the available lithography resolution. The illustration of the grating structure can be found in the next section.

Since the slit and the grating are in the same plane (see figure 2) and thus can be fabricated on the same wafer, spectrometer assembly does not involve the alignment of the slit relative to the grating. Only the opposing members need to be laterally aligned and set for gap spacing. The alignment tolerances for this part were calculated to be $100 \mu \mathrm{m}$ for the lateral alignment and $15 \mu \mathrm{m}$ for the distance to the lower plate. Normally the grating diffracts light into several diffraction orders, but only one order is imaged on the photosensor. That is why it is important to direct most of the light energy into the imaged diffraction order (usually first order) to achieve a better signal-to-noise ratio.

The ratio of the light energy in the specified diffraction order to the energy falling on the grating is generally referred to as the grating efficiency in that order. The grating efficiency is increased by an optimized groove profile. The most effective profile is triangular with the slope angle of the groove facets providing the reflection of the incident light in the direction of the specified diffracted order. Such gratings are called blazed gratings and the corresponding angle is the blaze angle. However the lithographic technique does not allow fabrication of the blazed gratings, the only physical parameter of the grating that can be optimized is the depth of the grooves. The dependence of the grating efficiency on the groove depth can be calculated numerically. Finally, for the grating of the designed spectrometer operating in the first diffraction order the groove depth was selected to be $230 \mathrm{~nm}$, which provides maximum diffraction of about $38 \%$ at the wavelength of $630 \mathrm{~nm}$ and minimum efficiency of $29 \%$ at $680 \mathrm{~nm}$ for an Al-coated grating in the $600-700 \mathrm{~nm}$ wavelength range.

\section{Fabrication and characterization of the spectrometer}

All elements of the spectrometer, including the slit and the grating, were fabricated on a single Al-coated glass wafer. The wafer was covered with $650 \mathrm{~nm}$ of aluminum and then several process steps followed. First, the grating patterns were

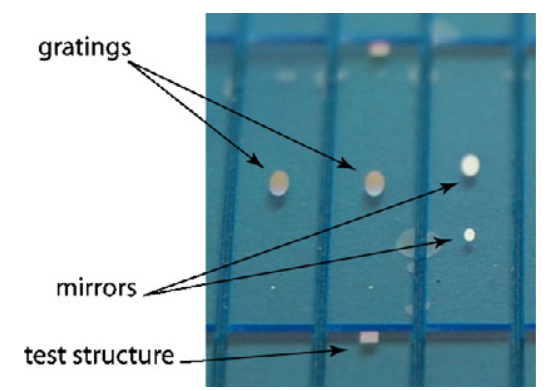

Figure 3. Diced wafer containing the spectrometer parts: elliptical grating and mirrors.

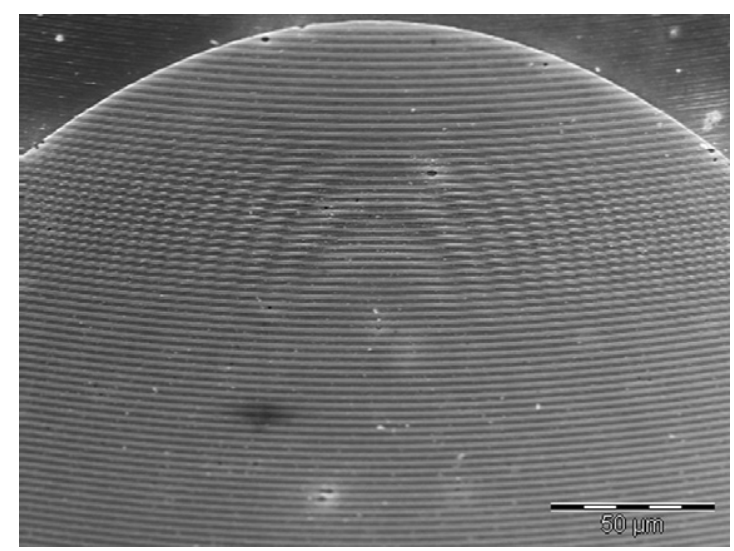

Figure 4. SEM photograph of part of the diffraction grating.

etched by plasma, to form about $230 \mathrm{~nm}$ deep grooves. Since the width of the grooves was small it could be difficult to measure the etched depth in aluminum and to control the etching process. That is why a test structure consisting of lines of $10 \mu \mathrm{m}$ width was included to facilitate etch-depth measurement. Subsequently, photolithography was applied for patterning of the aluminum to define the input slit, the grating and the mirrors. The last process step was dicing of the wafer. Figure 3 presents a photograph of the part of the diced wafer that contains elliptical mirrors and gratings. Also the rectangular test structure is clearly visible.

The fabricated gratings were examined using a scanning electron microscope (SEM). The SEM photos of a large part of the grating and its edge are presented in figures 4 and 5 , respectively. Figure 4 illustrates the structure of the curved grating grooves. Note that the concentric circles that are visible in the central part of figure 4 are not due to a grating defect, but produced by a Moiré effect in the SEM imaging system.

Figure 5 reveals that the shape of the grooves is not rectangular, which is due to the rather narrow width of the grooves.

The groove width was at the limit of the lithography resolution and thus the images of the lines on the photoresist, which determines the pattern to be etched, were blurred. This effect resulted in the rounding of the grooves edges during the etching process. Such rounded edges do not influence the imaging properties of the grating; however, the diffraction 


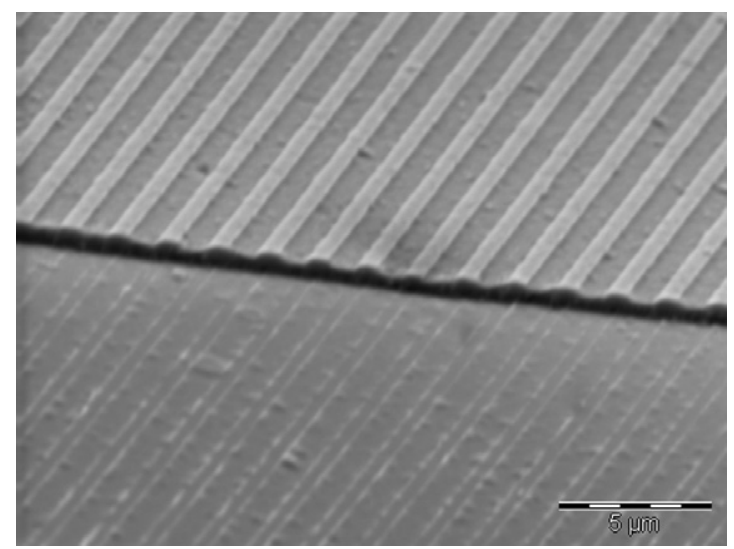

Figure 5. SEM photograph of the edge of the diffraction grating.

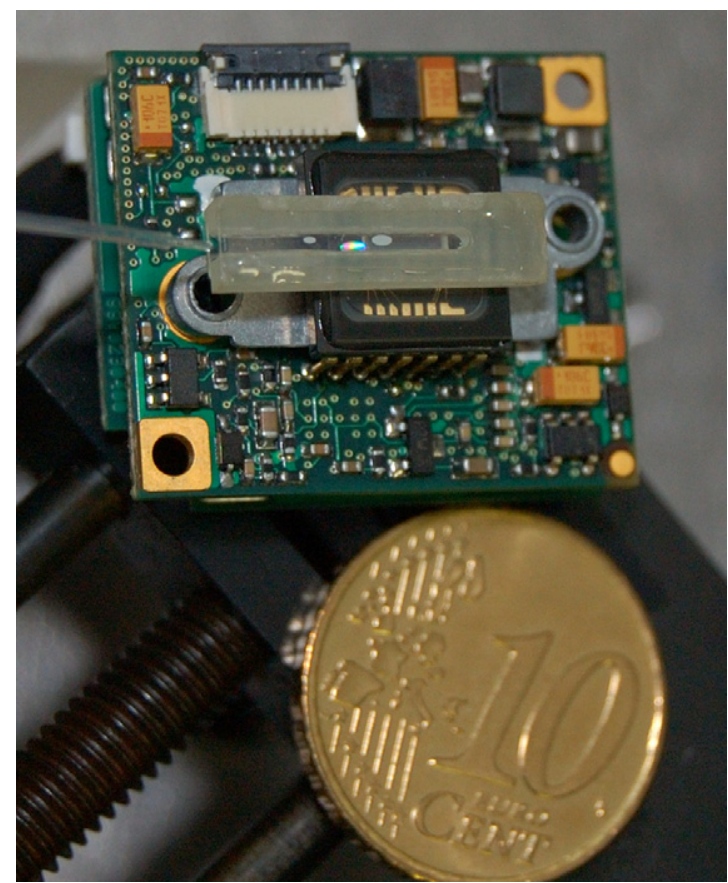

Figure 6. Assembled spectrometer mounted on top of the CCD image sensor.

efficiency can be reduced. Measurements of the grating efficiency were performed at a wavelength of $632 \mathrm{~nm}$ using a He-Ne laser and demonstrated an efficiency of $35 \%$ in the first diffraction order.

After dicing of the wafer the two parts of the spectrometer were assembled and mounted on top of the CCD image sensor. The completed microspectrometer is shown in figure 6 .

Due to relaxed requirements for alignment of the glass plates it was possible to use $1.5 \mathrm{~mm}$ thick spacer structures machined at the mechanical workshop for assembly. The spacer was fabricated with special directing grooves to facilitate lateral alignment of the glass pieces. Thus the glass plates were simply glued to the spacer and formed a ready for use device. A multi-mode optical fiber was used to feed the

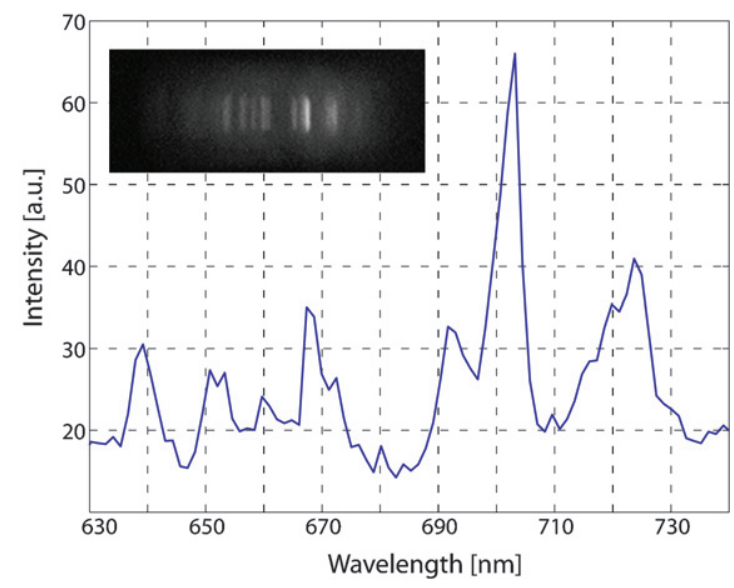

Figure 7. Ne spectral pattern captured with a CCD camera and graphical representation of the Ne spectrum.

input light to be spectrally analyzed by the spectrometer. One of the cleaved fiber tips was exposed to a light source under test and the second fiber tip illuminated the input slit of the spectrometer.

As an example of device performance, figure 7 shows the spectral pattern of Ne captured with the CCD camera and the graphical representation of the spectrum in the range of 630-730 nm, and also reveals the presence of stray light. The glass surface around the input slit at the angle of incidence of $60^{\circ}$ reflects about $9 \%$ of the light falling on the input slit due to Fresnel reflection. Thus spectral image presented in figure 7 is a superposition of the dispersed images of the entrance slit and the fiber tip that was used for illumination of the slit. Such elliptical images of the fiber tip can also be recognized in the inset of figure 7 . The stray light actually is a part of the instrumental function of the spectrometer. The elimination of Fresnel reflections would substantially decrease the level of stray light, which can be achieved by deposition of an appropriate coating on the unused glass surfaces. The coating process would involve one additional lithography step: the deposition of a light-absorbing material on top of a wafer, followed by the selective removal of this material from the aluminum grating areas, mirrors and the part of the glass plate covering the imaging sensor. Implementation of such a coating is the scope of our future work.

The spectrometer resolution can be estimated measuring the width of a monochromatic line presented in figure 7 . For example accounting for the 10 au. step in figure 7 the full height of the $703 \mathrm{~nm}$ spectral line is about $65 \mathrm{au}$, so the line width should be measured at about 37 au. Finally, the resolution of the spectrometer was estimated to be $6 \mathrm{~nm}$ at the wavelength of $703 \mathrm{~nm}$ and $4 \mathrm{~nm}$ in the center of the operating bandwidth (near $650 \mathrm{~nm}$ ), which differs, but not significantly, from the model estimates.

\section{Conclusions}

The design of the microspectrometer was based on analytical modeling. Practical constraints forced to a configuration 
that slightly departs from the model optimum. Although a maximum angle of incidence yields the best resolution, Fresnel reflections force toward a design with an angle of incidence limited to $60^{\circ}$. Moreover, the planarity of the design results in an image sensor mounted in parallel to the grating plane. With these constraints the FWHM resolution of the designed spectrometer was predicted to be about $7 \mathrm{~nm}$.

The planar design with two opposing members simplifies spectrometer fabrication and assembly considerably. The highly miniaturized (dimensions only $11 \times 1.5 \times 3 \mathrm{~mm}^{3}$ ) spectrometer demonstrated a FWHM spectral resolution of $6 \mathrm{~nm}$ within a $100 \mathrm{~nm}$ operating range, which is in good agreement with the analytical predictions. A spectrograph with such parameters can be used for example in paper or cosmetic industry (colorimetry) and for LED characterization. The planar approach enables the integration of several gratings designed for different spectral regions on the same chip. Thus the operating bandwidth of such an arrayed spectrometer can be increased. MEMS compatible fabrication offers potential for high production volume, which would allow for a highly competitive solution in a high-volume application.

Improving resolution is possible if the design or technology constraints are relaxed. One constraint is the input aperture, which is already small. The further reduction of the input aperture can be considered if the diffraction efficiency of the grating can be increased to compensate for such loss in optical throughput or if the light sources to be measured are sufficiently bright to produce enough signals with such a reduced aperture. The most important constraint is the one for the incident angle resulted from Fresnel reflection. If such reflections are eliminated using an antireflection coating the incident angle can be increased to the optimal value of about $76^{\circ}$ resulting in the improvement of spectral resolution by a factor 3, which implies that a FWHM resolution of about $2 \mathrm{~nm}$ is possible. Therefore, future work toward the improvement of the planar spectrometer is focused on the elimination of the Fresnel reflections from the unused surfaces of the device and on the IC-compatible fabrication of more efficient diffraction gratings.

\section{Acknowledgments}

The authors acknowledge financial support from STW, The Dutch Technology Foundation, grant DET 6667. They are thankful to Lina Sarro and Charles de Boer (DIMES, TU Delft) for the very useful discussions and wafer processing and to Jan Groeneweg and Jan Cornelis Wolff (DIMES, TU Delft) for the mask fabrication.

\section{References}

[1] Wolffenbuttel R F 2005 MEMS-based optical mini- and microspectrometers for the visible and infrared spectral range J. Micromech. Microeng. 15 S145-52

[2] Kwa T A and Wolffenbuttel R F 1992 Integrated grating/detector array fabricated in silicon using micromachining techniques Sensors Actuators A 31 259-66

[3] Kong S H and Wolffenbuttel R F 2005 Spectral performance of a micromachined infrared spectrum analyzer in silicon IEEE Trans. Instrum. Meas. 54 264-7

[4] Yen H W, Friedrich H R, Morrison R J and Tangonan G L 1981 Planar Rowland spectrometer for fiber-optic wavelength demultiplexing Opt. Lett. 6 639-41

[5] Sander D and Muller J 2001 Selffocusing phase transmission grating for an integrated optical microspectrometer Sensors Actuators A 88 1-9

[6] Avrutsky I, Chaganti K, Salakhutdinov I and Auner G 2006 Concept of a miniature optical spectrometer using integrated optical and micro-optical components Appl. Opt. 45 7811-7

[7] Ura S, Okayama F, Shiroshita K, Nishio K, Sasaki T, Nishihara H, Yotsuya T, Okano M and Satoh K 2003 Planar reflection grating lens for compact spectroscopic imaging system Appl. Opt. 42 175-80

[8] Ura S, Sasaki T and Nishihara H 2001 Combination of grating lenses for color splitting and imaging Appl. Opt. 40 5819-24

[9] Kostuk R K, Goodman J W and Hesselink L 1987 Design considerations for holographic optical interconnects Appl. Opt. 26 3947-53

[10] Noda H, Namioka T and Seya M 1974 Geometric theory of the grating J. Opt. Soc. Am. 64 1031-6

[11] Palmer C and McKinney W R 1994 Imaging theory of plane-symmetric varied line-space grating systems $O p t$. Eng. 33 820-9 